\title{
Tecnura
}

\section{Fractura de roca andesita y análisis espectral de señales de emisión acústica}

\section{Andesite rock fracture and spectral analysis of acoustic emission signals}

\author{
Dino Alberto Filipussi ${ }^{1}$, Juan Pablo Muszkats², Miryam Patricia Sassano ${ }^{3}$, \\ Miguel Eduardo Zitto ${ }^{4}$, Rosa Piotrkowski ${ }^{5}$
}

Fecha de recepción: 12 de octubre de 2018

Fecha de aceptación: 15 de abril de 2019

Cómo citar: Filipussi, D., Muszkats, J., Sassano, M., Zitto, M., Piotrkowski, R. (2019). Fractura de roca andesita y análisis espectral de señales de emisión acústica. Tecnura, 23(61), 45-56. doi: https://doi.org/10.14483/22487638.15361

\section{Resumen}

Contexto: En las rocas sometidas a esfuerzos se producen señales de emisión acústica (EA). Esta y la información sísmica son complementarias tanto en sus aplicaciones como en su base teórica. Por ende, el estudio de frecuencias características en las señales de emisión acústica tiene relevancia para comprender la propagación de ondas sísmicas en la Tierra.

Método: Se realizó un ensayo de compresión uniaxial en probetas cilíndricas de roca andesita, Ilevadas hasta la rotura. Se registraron las señales de EA obtenidas durante el ensayo. Un análisis de las frecuencias de estas señales cabe en el contexto de un modelo elemental de foco sísmico, el cual describe el avance y la propagación de ondas durante la fractura de un material frágil. A partir de los espectros de Fourier de las señales, convenientemente representados en escala logarítmica, se obtiene la frecuencia esquina. Este parámetro da cuenta del tamaño de las fisuras durante el avance del daño en el material sometido a esfuerzo.

Resultados: En un primer análisis se determinó la frecuencia esquina. En un segundo análisis, se filtraron las señales para distintas bandas de frecuencias con transformada Wavelet y se obtuvo el Wavelet Energy b-value (bWE) correspondiente.

Conclusiones: El estudio de la evolución del bWE para las distintas bandas de frecuencia permitió determinar frecuencias características que se pueden asociar a distintos mecanismos de fracturas en las rocas.

Palabras clave: emisión acústica, frecuencia esquina, roca andesita, transformada Wavelet, b-value.

\section{Abstract}

Context: Stressed rocks produce acoustic emission signals. Acoustic emission and seismic information are complementary in their applications as well as

1 Licenciado en Ciencias Físicas, doctor en Ingeniería. Profesor en la Escuela de Ciencia y Tecnología, Universidad Nacional de San Martín. Comisión Nacional de Energía Atómica, Centro Atómico Constituyentes, Departamento ICES. Ciudad Autónoma de Buenos Aires, Argentina. Contacto: filipuss@ @nea.gov.ar ORCID: https://orcid.org/0000-0002-9585-3043

2 Licenciado en Matemática, magíster en Ingeniería Matemática. Profesor adjunto, Universidad Nacional del Noroeste de la Provincia de Buenos Aires. Junín, Argentina. Contacto: jpmuszkats@comunidad.unnoba.edu.ar ORCID: https://orcid.org/0000-0001-8176-558X

3 Licenciada en Matemática, magíster en Ingeniería Matemática. Profesora adjunta, Facultad de Ingeniería, Universidad de Buenos Aires. Ciudad Autónoma de Buenos Aires, Argentina. Contacto: psassano@fi.uba.ar ORCID: https://orcid.org/0000-0002-5802-4756

4 Licenciado en Ciencias Físicas, magíster en Simulación Numérica y Control. Profesor adjunto, Facultad de Ingeniería, Universidad de Buenos Aires. Ciudad Autónoma de Buenos Aires, Argentina. Contacto: mzitto@fi.uba.ar ORCID: https://orcid.org/0000-0002-6423-1719

5 Licenciada en Ciencias Físicas, doctora en Ciencias Físicas. Centro de Matemática Aplicada, Universidad Nacional de San Martín. Profesora consulta asociada, Facultad de Ingeniería, Universidad de Buenos Aires. Ciudad Autónoma de Buenos Aires, Argentina. Contacto: rosap46@ gmail.com ORCID: https://orcid.org/0000-0002-6004-5679 
on their theoretical basis. Thus, studying characteristic frequencies in acoustic emission signals is relevant to understanding the seismic wave propagation on the Earth.

Method: A uniaxial compression test was performed on cylindrical samples of andesite rock, which were stressed up to breakage. Acoustic emission signals were recorded during the test. Frequency analysis in these signals may be carried in the context of an elementary seismic focus model. This model describes the fracture advance and propagation during brittle material fracture. The corner frequency is obtained from the Fourier spectrum of the signals, conveniently represented in log scale. This parameter accounts for the size of fissures as damage progresses when the material is stressed.

Results: The corner frequency was determined on a first analysis. On a second analysis, the signals were filtered on different bands with the Wavelet Transform and the corresponding wavelet energy b-value was obtained.

Conclusions: Studying the evolution of the wavelet energy b-value in different frequency bands allowed the determination of characteristic frequencies which can be associated with different fracture regimes in rocks.

Keywords: acoustic emission, corner frequency, andesite rock, wavelet transform, b-value.

\section{INTRODUCCIÓN}

En el interior de los materiales sometidos a esfuerzos se generan ondas elásticas producidas por la deformación del material y otras fuentes, como las debidas al crecimiento de fisuras, movimiento de dislocaciones, rotura de inclusiones, etc. La técnica de emisión acústica (EA) permite obtener información de estos procesos, detectar y medir estas ondas con sensores piezoeléctricos que transforman la señal mecánica en señal eléctrica. Las ondas elásticas provenientes del interior del material son registradas sobre la superficie, donde se ubican los sensores piezoeléctricos. Estas señales, en la forma de ráfagas Ilamadas hits, son procesadas para su posterior análisis. La señal eléctrica de EA es de muy baja amplitud $(10 \mu \mathrm{V})$ y de alta frecuencia $(50 \mathrm{kHz}$ a $1 \mathrm{MHz})$. Como se ilustra en la figura 1, exhibe eventos discretos con forma de pulsos oscilantes que alcanzan una amplitud máxima en un tiempo de subida (rise time) de la señal, para luego amortiguarse.

El equipo de EA (figura 2) cuenta con una placa electrónica que se encarga de la toma de las amplitudes de la señal en función del tiempo, con lo cual es posible observar la forma de onda de la señal; más tarde la placa procesa esta información y computa los valores de los parámetros característicos de la señal: energía, valor cuadrático medio (valor RMS), amplitud, rise time, duración del evento, etc. Esta técnica de EA proporciona un método para caracterizar los daños que sufre el material cuando es llevado a la rotura y dar cuenta, entre otros, de los procesos de microfisurado, crecimiento de fisuras y, por último, la coalescencia en macrofisuras hasta la rotura final del material.

Desde la década de 1960 se registran artículos sobre fractura de rocas sometidas a compresión (Hoek y Bieniawski, 1965; Lockner, 1993; Scholz, 1968) y es también un tema de actualidad por sus aplicaciones (Mirmohammadlou, Memarian, Mohammadi y Jafari, 2017). Resulta interesante observar que la EA es generada en distintas escalas espaciales y temporales abarcando desde la ruptura de ligaduras atómicas hasta fallas sísmicas. Por ello, la información sísmica y la EA son complementarias, tanto en sus aplicaciones como en su base teórica. A partir de esta idea, para dar cuenta de los procesos de daño en un material rocoso sometido a esfuerzos, hay trabajos (Carpinteri, Lacidogna y Puzzi, 2009; Rao y Prasanna Lakshmi, 2005; Shiotani, Yuyama, Li, y Ohtsu, 2001; Zhang, 


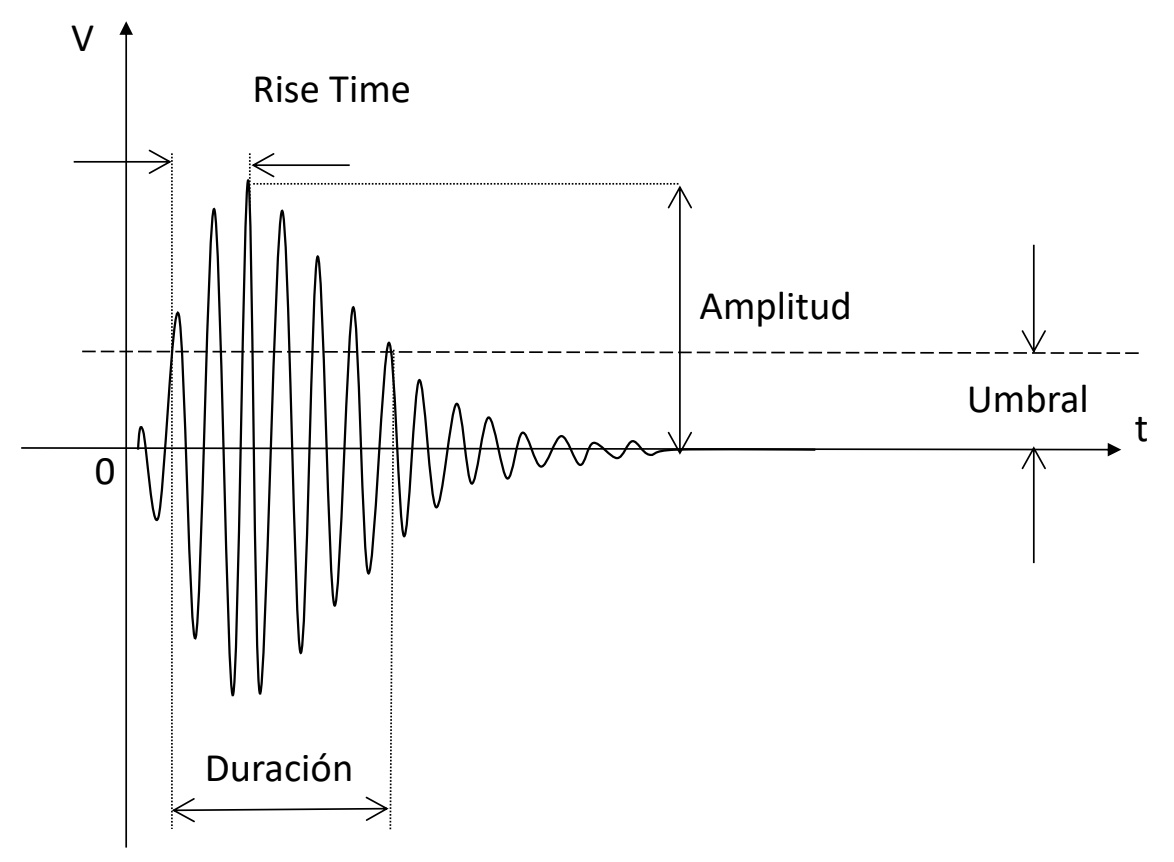

Figura 1. Señal de EA y parámetros que la caracterizan

Fuente: elaboración propia.

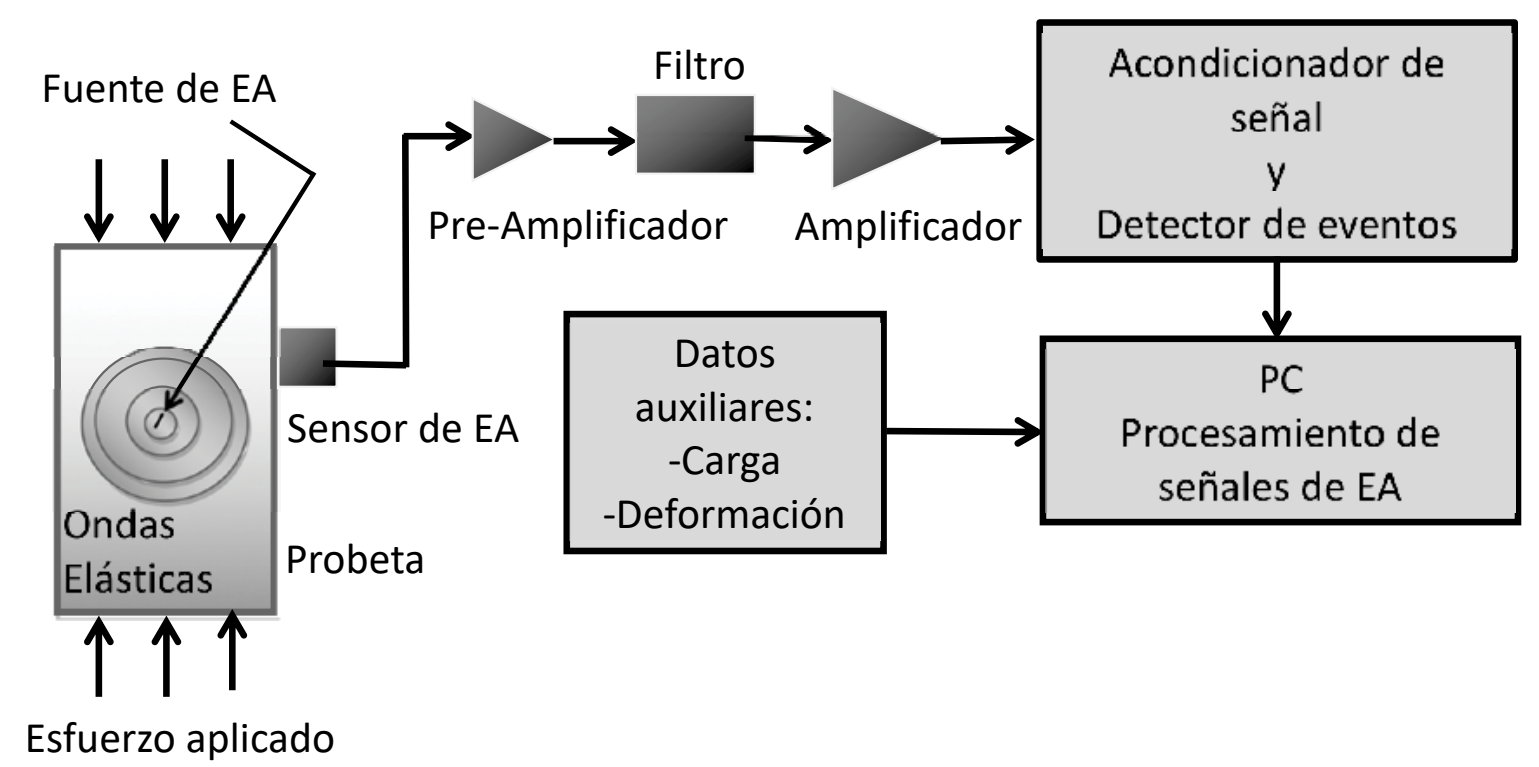

Figura 2. Equipo de EA. Adquisición y procesamiento de datos

Fuente: elaboración propia.

2018) que estudian el valor del $b$-value. Este parámetro surge de la distribución del número acumulado de hits de EA en función de su respectiva amplitud. Se comprueba que la relación en escala log-log es lineal y es conocida su aplicación en la sísmica como ley de Gutenberg-Richter. 


\section{METODOLOGÍA}

\section{Diseño experimental}

Siguiendo el trabajo de Filipussi et al. (2015), se ensayaron cuatro probetas cilíndricas de $75 \mathrm{~mm}$ de diámetro y $150 \mathrm{~mm}$ de largo, sobre testigos de andesita extraídos in situ de roca del Cerro Blanco, provincia de San Juan, Argentina. Estas probetas fueron ensayadas a compresión simple uniaxial y llevadas a la rotura. Se utilizó una máquina marca CGTS de 100 toneladas de capacidad, de tipo servohidráulica y lazo cerrado donde se impuso una velocidad de desplazamiento de actuador de 0,12 $\mathrm{mm} / \mathrm{min}$. La figura 3 muestra el arreglo experimental de una probeta de andesita instrumentada con sensores de EA.

Se emplearon tres sensores piezoeléctricos para monitorear la EA. En la superficie de la probeta se colocaron dos sensores, uno sintonizado a $60 \mathrm{kHz}$ y otro a $150 \mathrm{kHz}$. Al tercero, de banda ancha, se lo adhirió al plato de carga inferior teniendo en cuenta la propiedad de buena guía de onda del metal involucrado. El equipo de EA se completó con una placa de dos canales de (Physical Acoustic Corporation) y se utilizó el software comercial AEWIN para el análisis y determinación de la mayoría de los parámetros característicos de las señales.

\section{Modelo de fractura. Frecuencia esquina}

D. Filipussi, Piotrkowski, y Ruzzante (2012) simulan una señal de EA cuya fuente es una fisura que se propaga en un medio material frágil, homogéneo e isótropo, a partir de un modelo de fractura cuyos fundamentos provienen de la geofísica (Aki y Richards, 1980). La duración del evento de EA, su amplitud y tiempo de subida son estimados por el modelo y se relacionan con características de la fuente. El análisis del espectro de Fourier de la señal de EA obtenida del modelo está dado por la ecuación (1).

$$
|\widehat{\Omega}(\omega)|=\frac{U_{0} A}{\sqrt{1+\omega^{2} T}}\left|\frac{\operatorname{sen}\left(\frac{\tau_{0} \omega}{2}\right)}{\frac{\tau_{0} \omega}{2}}\right|
$$

Donde, es la transformada de Fourier del campo de desplazamiento; Uo, el valor máximo alcanzado por la discontinuidad del desplazamiento en la fractura; $A$, el área de la fractura; $T$, el tiempo en

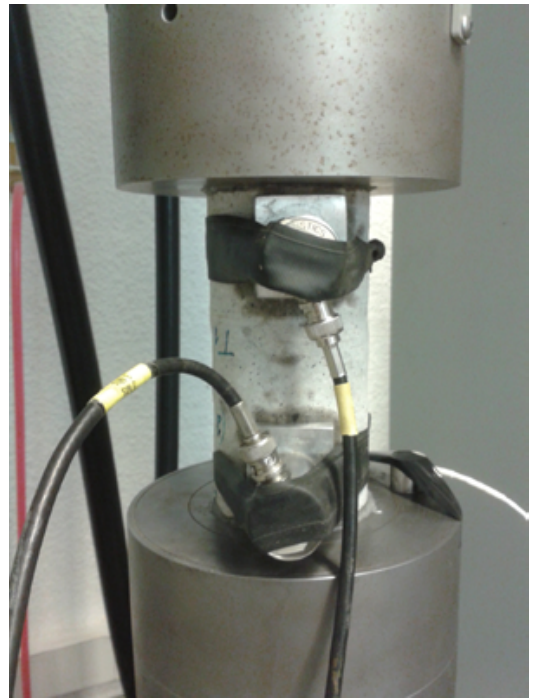

Figura 3. Configuración experimental y equipo de medición de EA

Fuente: elaboración propia. 
alcanzar la discontinuidad un $63 \%$ de $U_{0}$, y $\tau_{o^{\prime}}$ el tiempo de subida de la señal a detectar. En el espectro teórico determinado por el modelo se observa que la señal es constante a bajas frecuencias y luego decrece a frecuencias más altas con la inversa del cuadrado de la frecuencia. La frecuencia de encuentro de estas dos tendencias del espectro en una escala log-log se denomina frecuencia esquina $f_{c}$. El modelo predice también que la longitud de la fractura es inversamente proporcional a la frecuencia esquina y está dada por la ecuación (2).

$$
L=\frac{v}{2 \pi f_{c}(1-\eta \cos \theta)}
$$

Donde $\widehat{\Omega}(\omega), L$ es la longitud de la fisura; $\eta=\frac{v}{c}$ es la proporción entre la velocidad de avance de la fractura y la velocidad de onda del sonido en el medio material ( $\eta$ vale cerca de 1 para un material frágil); $\theta$ es el ángulo de detección respecto a la línea de avance de la fractura.
En este trabajo se estimó la longitud de las fisuras con esta última ecuación, a través de la medición de las frecuencias esquinas de las señales adquiridas en los instantes de tiempo en que la energía del hit alcanza valores máximos relativos. Se consideró que $\eta=0.7$ por ser el material frágil, que la detección fue aproximadamente en la línea de avance de la fractura $\left(\theta=0^{\circ}\right)$ y que la velocidad de propagación de onda en la andesita es $c=4500 \frac{\mathrm{m}}{\mathrm{s}}$ como se menciona en Schön (2011).

En la figura 4 se muestra un hit y en la figura $5 \mathrm{su}$ correspondiente espectro en escala log-log, donde se indica la frecuencia esquina determinada por el punto de intersección de las tendencias a baja y alta frecuencia.

Los hits seleccionados para la obtención de la frecuencia esquina corresponden a aquellos intervalos de tiempo donde el valor cuadrático medio RMS de las señales alcanza un máximo relativo, como se muestra en la figura 6.

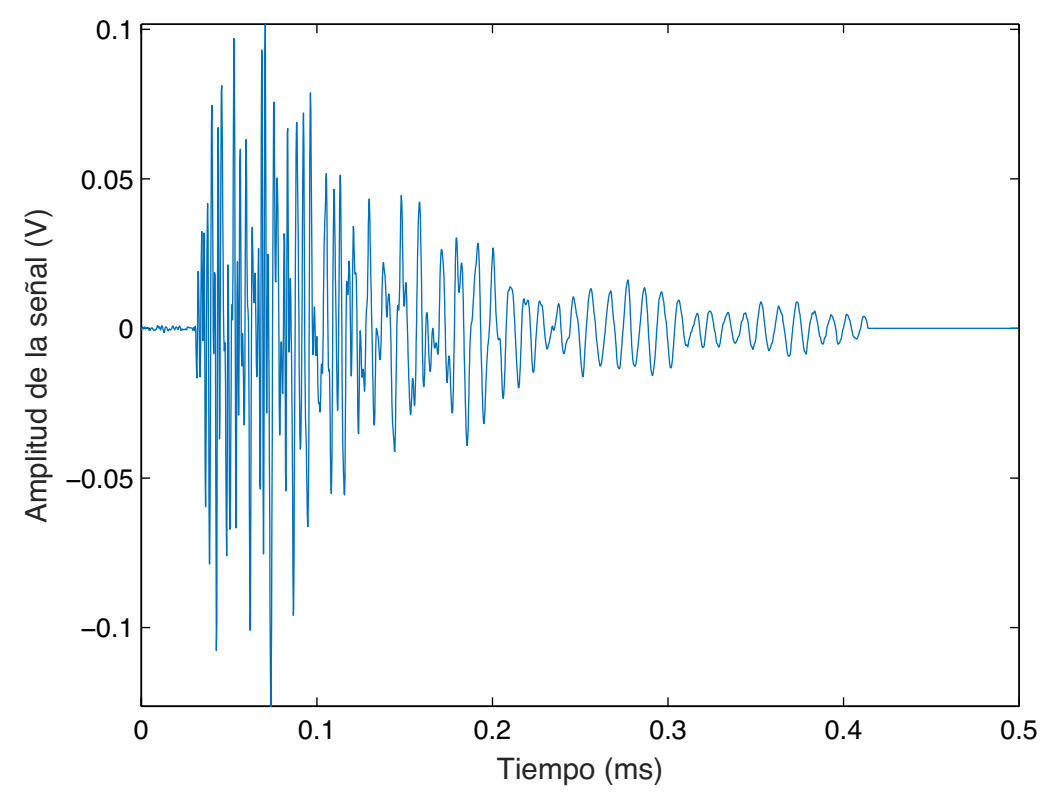

Figura 4. Gráfico de un hit

Fuente: elaboración propia. 


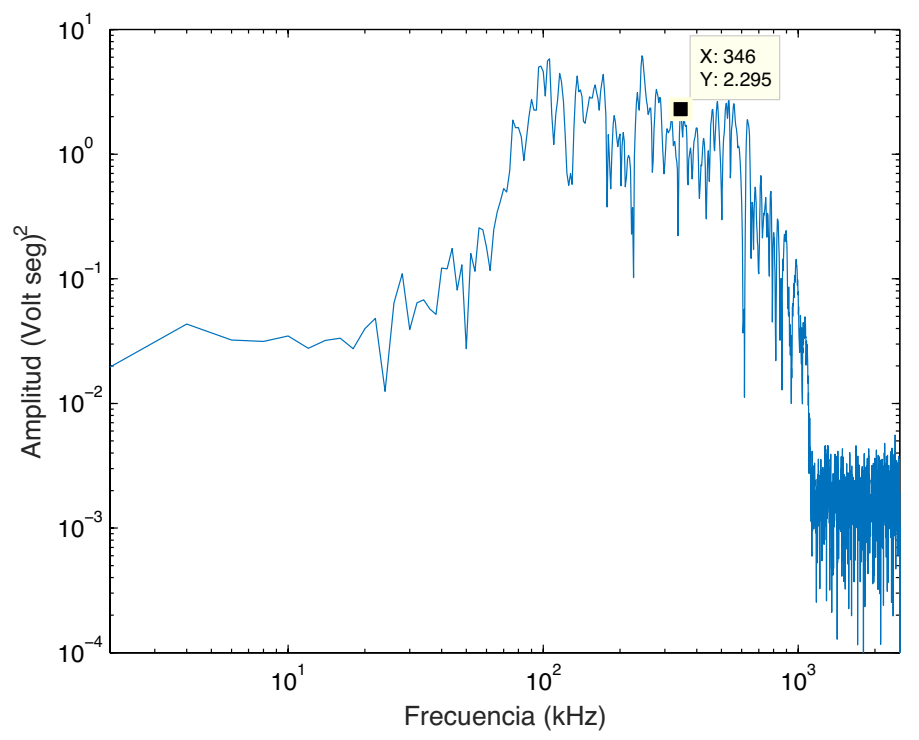

Figura 5. Espectro de Fourier de un hit en escala log-log con frecuencia esquina $302,1 \mathrm{kHz}$

Fuente: elaboración propia.

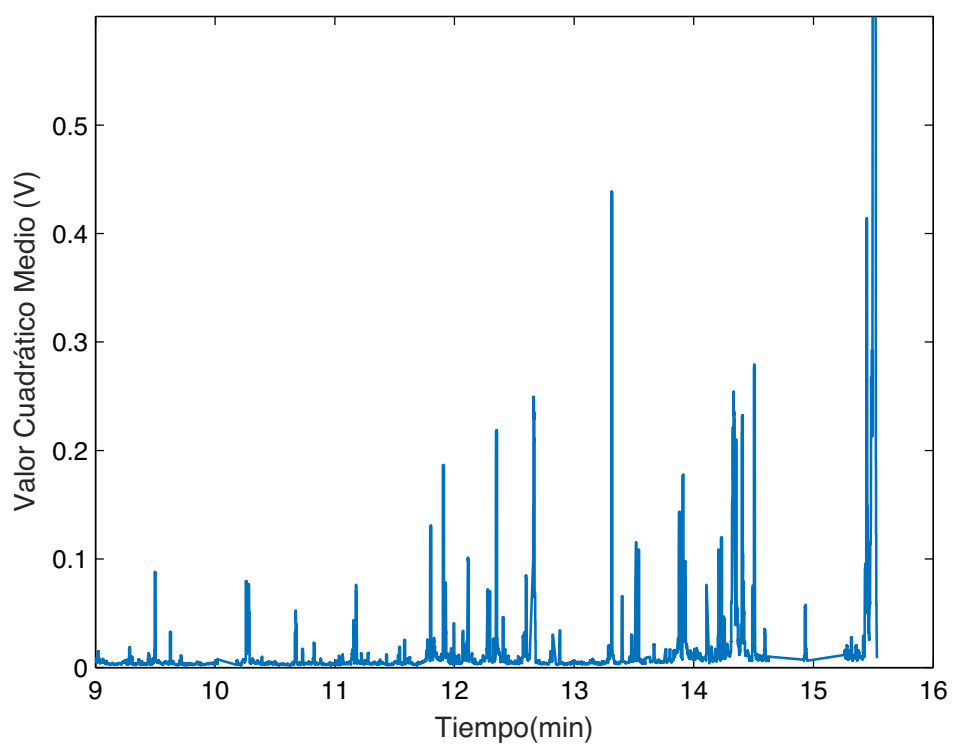

Figura 6. Valor cuadrático de cada hit en función del tiempo del ensayo

Fuente: elaboración propia.

La tabla 1 detalla los valores de frecuencia esquina determinados para los tiempos indicados que corresponden a un intervalo de tiempo que contiene a un máximo relativo de RMS. 
Tabla 1. Frecuencia esquina y su correspondiente longitud de fisura

\begin{tabular}{ccc}
\hline $\begin{array}{c}\text { Tiempo } \\
(\mathbf{s})\end{array}$ & $\begin{array}{c}\text { Frecuencia Equina } \\
(\mathbf{k H z})\end{array}$ & $\begin{array}{c}\text { Longitud de la fisura } \\
(\mathbf{m m})\end{array}$ \\
\hline 615,60 & 239,6 & 5,53 \\
\hline 615,74 & 302,1 & 4,39 \\
\hline 615,88 & 197,9 & 6,70 \\
\hline 616,02 & 270,8 & 4,89 \\
\hline 616,18 & 302,1 & 4,39 \\
\hline 616,34 & 354,2 & 3,74 \\
\hline 616,52 & 270,8 & 4,89 \\
\hline 616,73 & 291,7 & 4,54 \\
\hline 616,91 & 302,1 & 4,39 \\
\hline 617,18 & 291,7 & 4,54 \\
\hline
\end{tabular}

Fuente: elaboración propia.

\section{Transformada Wavelet continua}

La transformada wavelet continua (Continuous Wavelet Transform, CWT) permite, como la transformada de Fourier, estudiar las componentes de frecuencia de una señal. Además, brinda información acerca de la localización temporal de dichas componentes. Siguiendo la exposición de Boggess y Narcowich (2009), se considera una función Wavelet $\Psi(t)$ que cumpla con los requisitos de ser continua, tener decaimiento exponencial e integral nula en $\mathbb{R}$. Bajo estas condiciones, la CWT de una función $f \in L^{2}(\mathbb{R})$ es la función $W_{f}: \mathbb{R}^{2} \rightarrow \mathbb{C}$ definida por la ecuación (3).

$$
W_{f}(a, b)=\frac{1}{\sqrt{|a|}} \int_{-\infty}^{\infty} f(t) \overline{\psi\left(\frac{t-b}{a}\right)} d t
$$

En el caso en que $a \neq 0$, mientras que $W_{f}(0, b)=0$. La integral definida por la ecuación (3) compara la función $f(t)$ con la Wavelet $\Psi(t)$ después de un cambio de escala y una traslación temporal. De esta forma, el valor $W_{f}(a, b)$ mide la componente en frecuencia de la señal $f(t)$ proporcional a (1/a) y localizada en torno al tiempo $b$.

En el presente trabajo se utilizó la Wavelet de Morlet definida por la ecuación (4).

$$
\psi(t)=\pi^{-1 / 4} \cdot e^{6 i t} \cdot e^{-t^{2} / 2}
$$

Los detalles de implementación de la CWT para señales discretas pueden consultarse en el trabajo clásico de Torrence y Compo (1998). En la figura 7 se ilustra uno de los hits estudiados y en la figura 8 su correspondiente escalograma, es decir, un gráfico de la densidad de energía Wavelet correspondiente a cada tiempo y frecuencia.

A partir de la transformada Wavelet de una señal pueden filtrarse las frecuencias indeseadas eliminando los coeficientes correspondientes y reconstruyendo la señal. En la figura 9 se ilustra el hit anterior filtrado entre $240 \mathrm{kHz}$ y $300 \mathrm{kHz}$, y en la figura 10, su correspondiente escalograma.

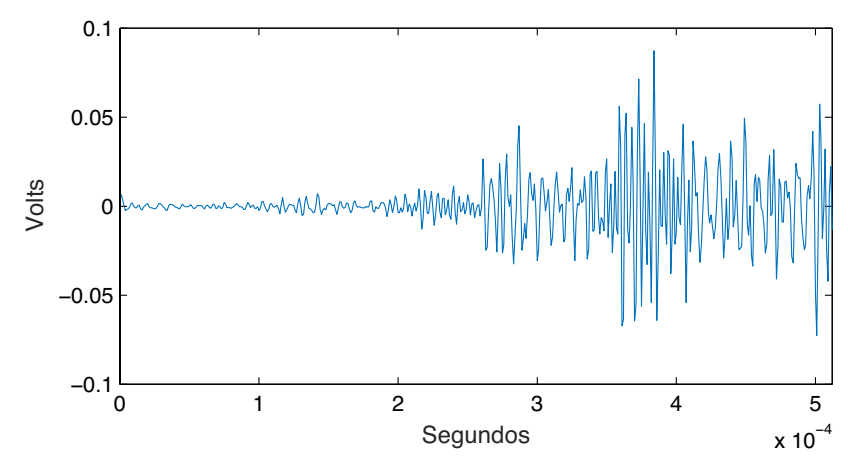

Figura 7. Gráfico de un hit

Fuente: elaboración propia. 


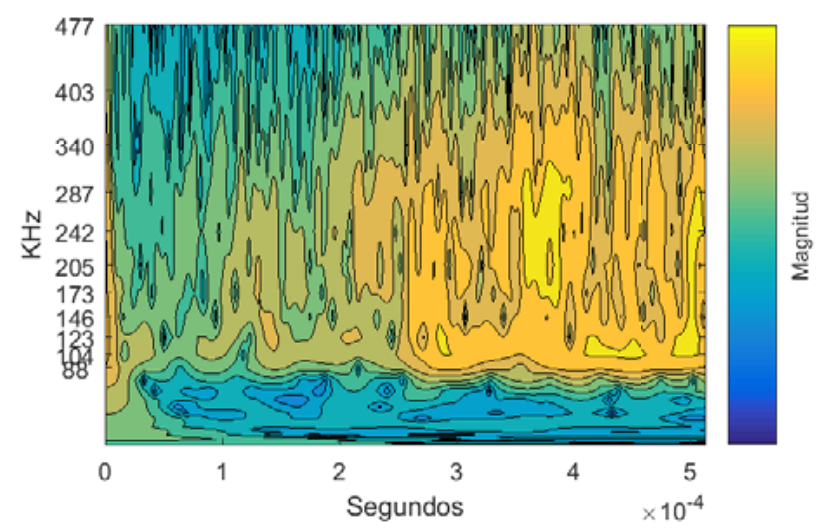

Figura 8. Escalograma de un hit

Fuente: elaboración propia.

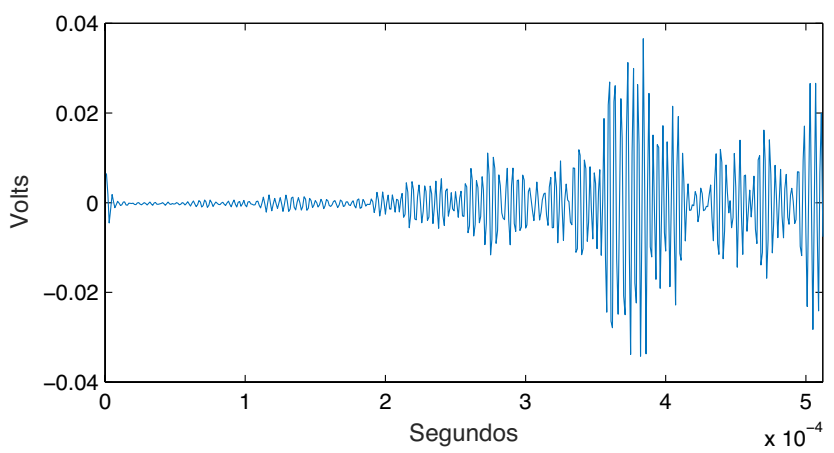

Figura 9. Gráfico de un hit filtrado

Fuente: elaboración propia.

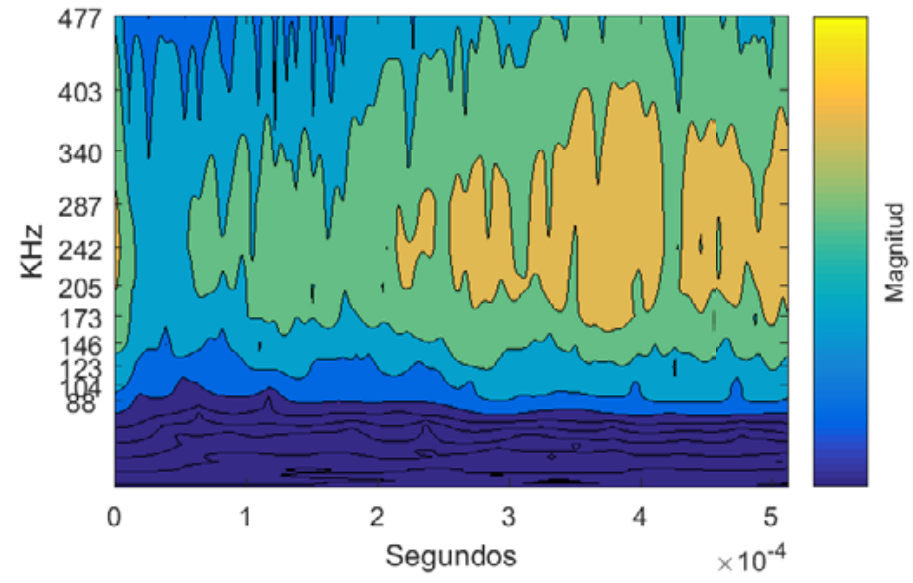

Figura 10. Escalograma de un hit filtrado

Fuente: elaboración propia 


\section{Aplicación al ensayo}

Al aplicar la CWT a cada hit registrado en el ensayo se conocen sus componentes de frecuencia localizadas en el tiempo. El gráfico de la figura 11 representa para cada hit la frecuencia de mayor energía. En el eje vertical se expresa la frecuencia en $\mathrm{kHz}$ y en el eje horizontal el tiempo real en segundos de ocurrencia del hit durante el ensayo. Se aprecia que las componentes de mayor frecuencia se manifiestan hacia el final del ensayo.

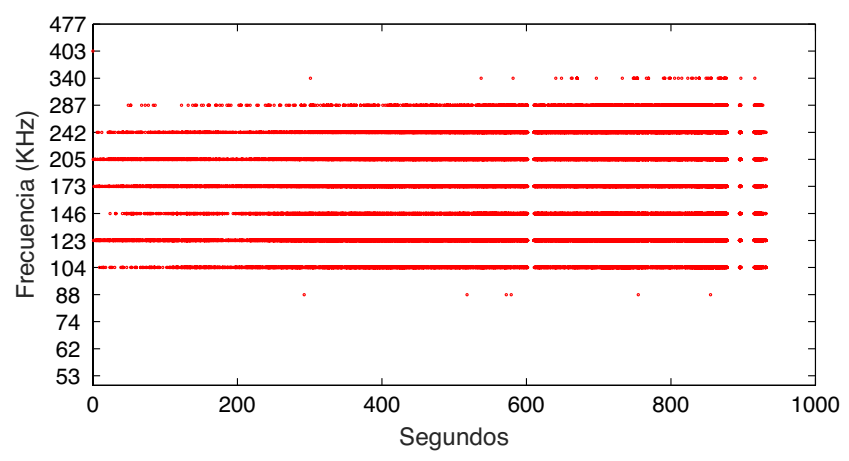

Figura 11. Frecuencia de máxima energía de cada hit

Fuente: elaboración propia.

Tabla 2. Conteo de hits a diferentes frecuencias

\begin{tabular}{cc}
\hline Frecuencia $\mathbf{( k H z )}$ & Cantidad de hits \\
\hline 88 & 6 \\
\hline 104 & 7066 \\
\hline 123 & 21386 \\
\hline 146 & 6589 \\
\hline 173 & 10373 \\
\hline 205 & 12769 \\
\hline 242 & 15603 \\
\hline 287 & 1527 \\
\hline 340 & 66 \\
\hline 403 & 1 \\
\hline
\end{tabular}

Fuente: elaboración propia.

Las dos frecuencias para las cuales se alcanza más veces el máximo de energía son $123 \mathrm{kHz}$ y $242 \mathrm{kHz}$. La figura 12 se obtuvo después de filtrar los hits para valores próximos a la frecuencia esquina: entre $240 \mathrm{kHz}$ y $300 \mathrm{kHz}$. Una vez más, se indica la frecuencia a la que cada hit alcanza su máxima energía. En esta ocasión, se agrega un círculo cuyo diámetro y color responden al nivel de energía alcanzado. Aunque no es concluyente, se aprecia cierta tendencia a que los hits más energéticos se den hacia el final del ensayo.

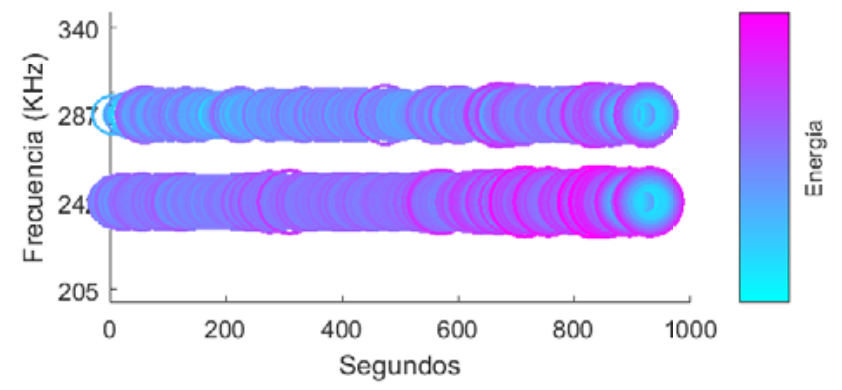

Figura 12. Frecuencias características luego de filtrar en base a la frecuencia esquina

Fuente: elaboración propia.

\section{Wavelet energy b-value}

Siguiendo a Sagasta et al. (2018), se presenta una adaptación de la ley de Gutenberg-Richter usada en sismología. En este contexto, la EA se interpreta como sismología de pequeña escala. Para ello se considera la energía wavelet $(A E E)$ de cada señal reconstruida luego del filtrado (ecuación (5)).

$$
A E E=\sum x_{i}^{2} \cdot \Delta t
$$

Luego se la vincula con la cantidad $N$ de hits cuya energía supera un valor dado de $A E E$. La figura 13 ilustra esta relación para la probeta del ensayo, con los hits reconstruidos luego del filtrado entre 240 kHz y 300 kHz. Para cada hit representado se asigna además un color que indica su ocurrencia en el tiempo. Una vez más se aprecia que los hits de mayor energía ocurren hacia el final del ensayo. 
Como sugiere el gráfico, se puede postular una relación lineal entre los logaritmos de estas variables (ecuación (6)).

$$
\log _{10} N(A E E)=a-b_{E} \cdot \log _{10}(A E E)
$$

El Wavelet Energy $b$-value (bWE) se define justamente como el valor $b_{E}$ de esta fórmula. Un valor grande de $b_{E}$ indica una rápida variación de $N$ con respecto a la energía, lo cual representaría un régimen de fractura estable. Esto es usualmente indicativo de una gran cantidad de eventos de energía relativamente baja, lo cual es consistente con procesos de creación de microfracturas o de crecimiento lento de fracturas. En cambio, un valor pequeño de $b_{E}$ indica

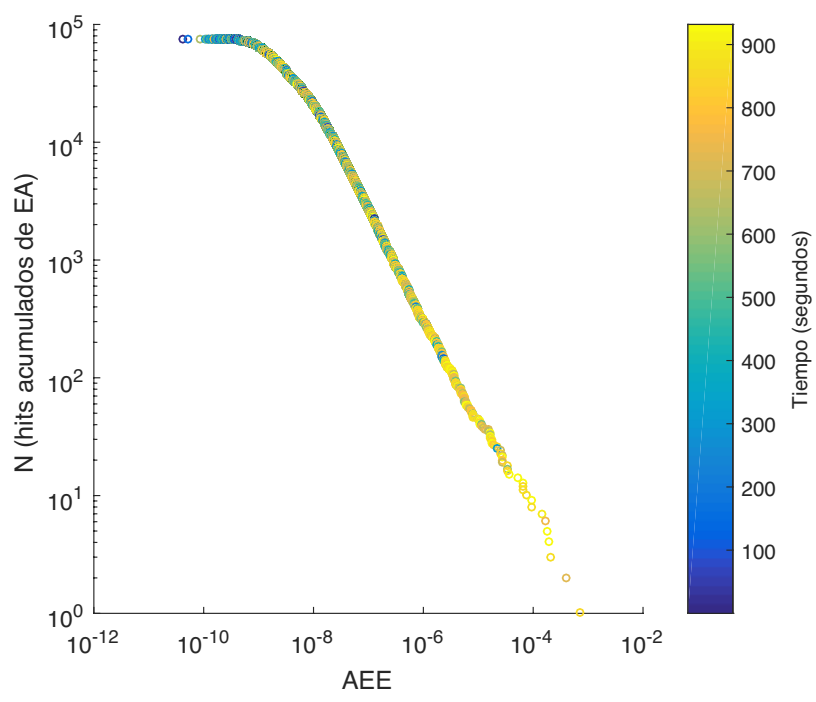

Figura 13. $N$ vs $A E E$

Fuente: elaboración propia.

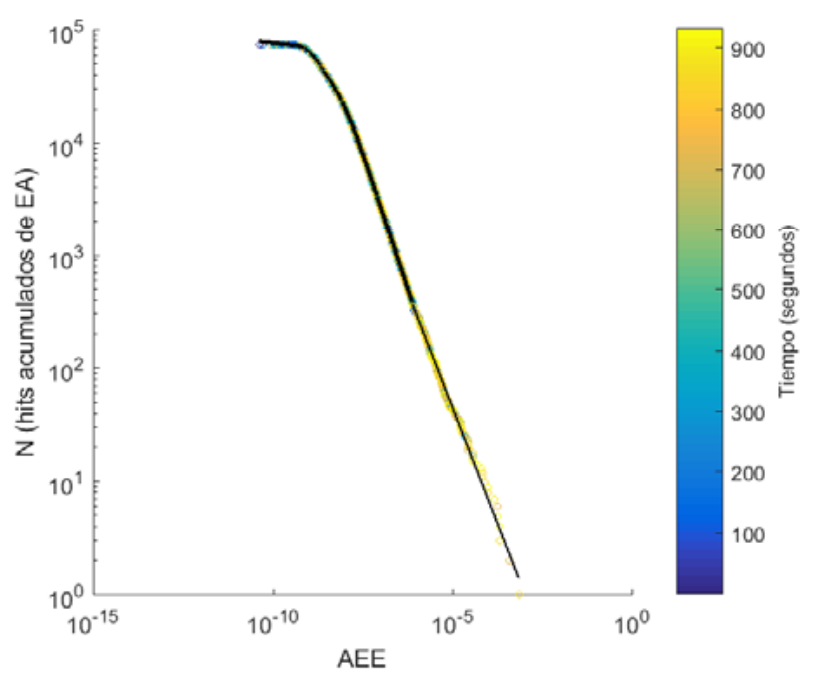

Figura 14. $N$ vs. AEE. Ajuste por cuadrados mínimos

Fuente: elaboración propia. 
una variación brusca de energía en pocos hits, lo que sugiere la formación de macrofracturas o crecimiento rápido de fracturas. Es importante identificar los valores de energía para los cuales se alcanzan mínimos locales del índice. La figura 14 ilustra las rectas de cuadrados mínimos obtenidas al dividir el intervalo de $A E E$ en subintervalos de igual cantidad de hits y calcular $b_{E}$ en cada uno de ellos. La figura 15 muestra el bWE obtenido para distintos subintervalos.

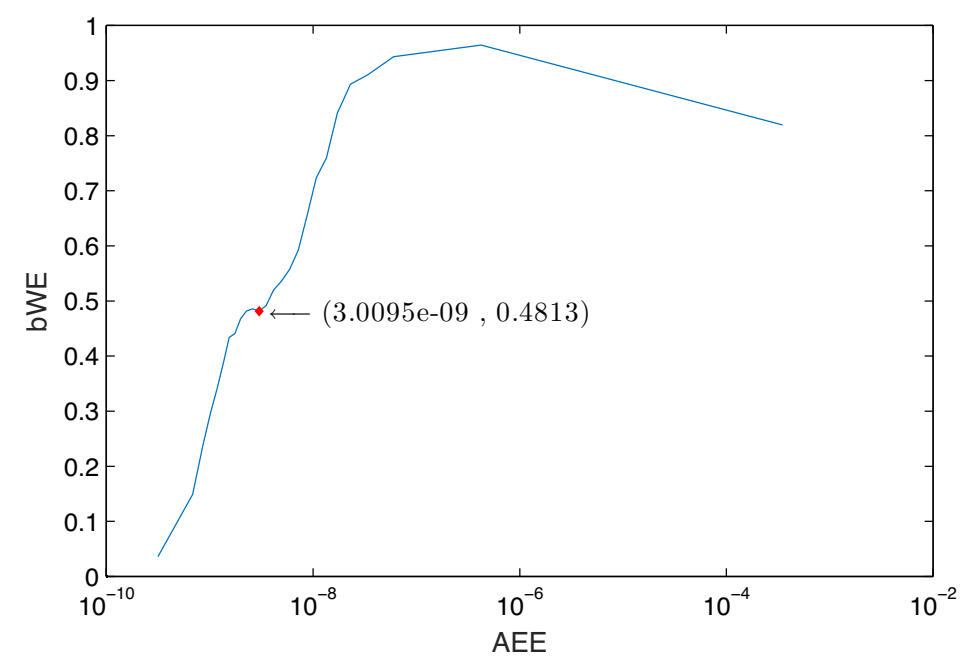

Figura 15. bWE obtenido para distintos subintervalos

Fuente: elaboración propia.

\section{RESULTADOS}

La frecuencia característica obtenida mediante la CWT resulta consistente con los resultados para la frecuencia esquina (ver tabla 2, donde se hace el conteo de hits a diferentes frecuencias).

El estudio de la evolución del bWE para las distintas bandas de frecuencia sugiere la existencia de energías características que se pueden asociar a distintos mecanismos de fracturas en las rocas. Los mínimos locales de bWE (figura 15) identifican estos valores críticos de AEE.

\section{CONCLUSIONES}

A partir de un ensayo de una probeta de andesita bajo compresión uniaxial llevada a la rotura y del registro de EA mediante un sensor de banda ancha, se pudo identificar en los hits de mayor energía una frecuencia esquina del orden de $300 \mathrm{kHz}$ que se correspondió con una longitud de fisura del orden de $5 \mathrm{~mm}$.

Los resultados obtenidos con el análisis Wavelet permiten una mayor precisión en la determinación de frecuencias características, que fueron $123 \mathrm{kHz}$ y $242 \mathrm{kHz}$, siendo esta última consistente con la frecuencia esquina. La combinación de ambos criterios, frecuencia esquina y máximo de energía de la CWT permitió, filtrando entre $240 \mathrm{kHz}$ y $300 \mathrm{kHz}$, encontrar con precisión las frecuencias de $242 \mathrm{kHz}$ y $287 \mathrm{kHz}$ que se podrían asignar a mecanismos de fractura. El bWE calculado en la banda $240-300 \mathrm{kHz}$ permitió hallar, mediante la localización de mínimos locales, valores críticos de energía que identificarían la aparición de macrofisuras. Los hits de mayor energía se manifiestan preponderantemente hacia el final del ensayo. 


\section{FINANCIAMIENTO}

Comisión Nacional de Energía Atómica, Centro Atómico Constituyentes, gerencia Desarrollo Tecnológico y Proyectos Especiales, Departamento ICES.

Este trabajo tiene apoyo financiero y es parte del Programa UBACyT 20020160100038BA.

\section{REFERENCIAS}

Aki, K. y Richards, P.G. (1980). Quantitative Seismology: Theory and Methods. Sausalito, CA: University Science Book. Recuperado de https://books.google. com.ar/books?id=k8R_QgAACAAJ

Boggess, A. y Narcowich, F.J. (2009). A first course in wavelets with Fourier analysis. Nueva Jersey: John Wiley \& Sons, Inc. doi: https://doi. org/10.1063/1.1485592

Carpinteri, A., Lacidogna, G. y Puzzi, S. (2009). From criticality to final collapse: Evolution of the "b-value" from 1.5 to 1.0. Chaos, Solitons and Fractals, 41(2), 843-853. doi: https://doi.org/10.1016/j. chaos.2008.04.010

Filipussi, D.A., Guzmán, C.A., Xargay, H.D., Hucailuk, C. y Torres, D.N. (2015). Study of Acoustic Emission in a Compression Test of Andesite Rock. Procedia Materials Science, 9, 292-297. doi: https://doi.org/10.1016/J.MSPRO.2015.04.037

Filipussi, D., Piotrkowski, R. y Ruzzante, J. (2012). Characterization of a Crack by the Acoustic Emission Signal Generated During Propagation. Procedia Materials Science, 1, 266-272. doi: https://doi.org/10.1016/j.mspro.2012.06.036

Hoek, E. y Bieniawski, Z.T. (1965). Brittle fracture propagation in rock under compression. International Journal of Fracture Mechanics, 1(3), 137-155. doi: https://doi.org/10.1007/BF00186851

Lockner, D. (1993). The role of acoustic emission in the study of rock fracture. International Journal of Rock Mechanics and Mining Sciences y
Geomechanics Abstracts, 30(7), 883-899. doi: https://doi.org/10.1016/0148-9062(93)90041-B

Mirmohammadlou, A., Memarian, H., Mohammadi, S. y Jafari, M. (2017). Experimental and Numerical Investigation of Rock Dynamic Fracture. International Journal of Mining and Geo-Engineering, 51(1), 37-46. doi: https://doi.org/10.22059/ IJMGE.2017.62151

Rao, M.V.M.S. y Prasanna Lakshmi, K.J. (2005). Analysis of b-value and improved b-value of acoustic emissions accompanying rock fracture. Current Science, 89(9), 1577-1582. doi: https://doi. org/10.2307/24110936

Sagasta, F., Zitto, M.E., Piotrkowski, R., Benavent-Climent, A., Suarez, E. y Gallego, A. (2018). Acoustic emission energy $b$-value for local damage evaluation in reinforced concrete structures subjected to seismic loadings. Mechanical Systems and Signal Processing, 102, 262-277. doi: https://doi.org/10.1016/j.ymssp.2017.09.022

Scholz, C.H. (1968). Microfracturing and the inelastic deformation of rock in compression. Journal of Geophysical Research, 73(4), 1417-1432. doi: https://doi.org/10.1029/JB073i004p01417

Schön, J. (2011). Physical properties of rocks: a workbook. Ámsterdam: Elsevier.

Shiotani, T., Yuyama, S., Li, Z.W. y Ohtsu, M. (2001). Application of AE improved b-value to quantitative evaluation of fracture process in concrete materials. Journal of Acoustic Emission, 19, 118-133.

Torrence, C. y Compo, G. (1998). A Practical Guide to Wavelet Analysis. Bulletin of the American Meteorological Society, 79(1), 61-78. doi: https://doi. org/10.1175/1520-0477(1998)079\&lt;0061:APGTWA\&gt;2.0.CO;2

Zhang, J. (2018). Investigation of Relation between Fracture Scale and Acoustic Emission Time-Frequency Parameters in Rocks. Shock and Vibration, 2018, 1-14. doi: https://doi.org/10.1155/2018/3057628

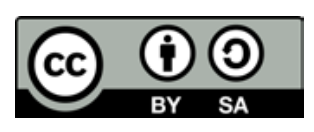

Tecnura • p-ISSN: 0123-921X • e-ISSN: 2248-7638 • Vol. 23 No. 61 • Julio - Septiembre de $2019 \bullet$ pp. 45-56 